\title{
Systematic geriatric assessment for older patients with frailty in the emergency department: a randomised controlled trial
}

\author{
Janne Alakare ${ }^{1 *}$, Kirsi Kemp1', Timo Strandberg ${ }^{2,3}$, Maaret Castrén ${ }^{1}$, Dimitrije Jakovljević ${ }^{4}$, Jukka Tolonen ${ }^{5}$ and \\ Veli-Pekka Harjola ${ }^{1}$
}

\begin{abstract}
Background: Comprehensive geriatric assessment provided in hospital wards in frail patients admitted to hospital has been shown to reduce mortality and increase the likelihood of living at home later. Systematic geriatric assessment provided in emergency departments (ED) may be effective for reducing days in hospital and unnecessary hospital admissions, but this has not yet been proven in randomised trials.

Methods: We conducted a single-centre, randomised controlled trial with a parallel-group, superiority design in an academic hospital ED.

ED patients aged $\geq 75$ years who were frail, or at risk of frailty, as defined by the Clinical Frailty Scale, were included in the trial. Patients were recruited during the period between December 11, 2018 and June 7, 2019, and followed up for 365 days.

For the intervention group, systematic geriatric assessment was added to their standard care in the ED, whereas the control group received standard care only.

The primary outcome was cumulative hospital stay during 365-day follow-up. The secondary outcomes included: admission rate from the index visit, total hospital admissions, ED-readmissions, proportion of patients living at home at 365 days, 365-day mortality, and fall-related ED-visits.
\end{abstract}

Results: A total of 432 patients, $63 \%$ female, with median age of 85 years, formed the analytic sample of 213 patients in the intervention group and 219 patients in the control group.

Cumulative hospital stay during one-year follow-up as rate per 100 person-years for the intervention and control groups were: 3470 and 3149 days, respectively, with rate ratio of 1.10 (95\% confidence interval, $0.55-2.19, P=.78$ ). Admission rates to hospital wards from the index ED visit for the intervention and control groups were: 62 and $70 \%$, respectively $(P=.10)$. No significant differences were observed between the groups for any outcomes.

Conclusion: Systematic geriatric assessment for older adults with frailty in the ED did not reduce hospital stay during one-year follow-up. No statistically significant difference was observed for any secondary outcomes. More coordinated, continuous interventions should be tested for potential benefits in long-term outcomes.

\footnotetext{
* Correspondence: janne.alakare@hus.fi

${ }^{1}$ Department of Emergency Medicine and Services, University of Helsinki and Helsinki University Hospital, PL 340 |Haartmaninkatu 4, 00029 HUS Helsinki, Finland

Full list of author information is available at the end of the article
}

(c) The Author(s). 2021 Open Access This article is licensed under a Creative Commons Attribution 4.0 International License, which permits use, sharing, adaptation, distribution and reproduction in any medium or format, as long as you give appropriate credit to the original author(s) and the source, provide a link to the Creative Commons licence, and indicate if changes were made. The images or other third party material in this article are included in the article's Creative Commons licence, unless indicated otherwise in a credit line to the material. If material is not included in the article's Creative Commons licence and your intended use is not permitted by statutory regulation or exceeds the permitted use, you will need to obtain permission directly from the copyright holder. To view a copy of this licence, visit http://creativecommons.org/licenses/by/4.0/. The Creative Commons Public Domain Dedication waiver (http://creativecommons.org/publicdomain/zero/1.0/) applies to the data made available in this article, unless otherwise stated in a credit line to the data. 
Trial registration: The trial was registered in the ClinicalTrials.gov (registration number and date NCT03751319 23/ 11/2018).

Keywords: Frailty, Aged, Aged, 80 and over, Emergency Departments, Geriatric Assessment, Length of Stay

\section{Introduction}

Frail older adults are a major patient group in emergency departments (ED). Many validated tools that have been developed to recognise and classify frailty are also implemented in ED care [1-4]. For example, the Rockwood Clinical Frailty Scale (CFS) has been validated with good inter-rater reliability for the ED-environment for predicting adverse outcomes. The CFS has been used in emergency care as a quick and feasible tool for frailty status assessment [5-10]. On the other hand, in-depth intervention, comprehensive geriatric assessment (CGA), when provided for admitted hospital patients, has been shown to be effective in reducing mortality and increasing the likelihood of subsequently living at home [11].

It is likely that many frail patients who visit EDs do not receive beneficial interventions which could be effective for long term outcomes. Possible reasons for this include: frailty may not be identified, patients may have multiple ED-visits with different or nonspecific complaints while geriatric syndromes remain disregarded, and organisations may not have services set up for geriatric care. Therefore, EDs may be a practical point for connecting geriatric care needs with a systematic approach.

In previous nonrandomised studies, physician-led CGA provided in acute care settings has been associated with reduced hospital admissions from the ED [12-15]; however, no efficacy for longer term outcomes has been shown. Nurse-led interventions provided after ED visits may be beneficial in preventing functional decline, but results concerning ED-readmissions and hospital admissions are mixed [16-25]. In our opinion, better understanding with the right scope and timing for geriatric interventions for frail older adults visiting EDs is required. We hypothesised that systematic, individualised, multi-dimensional geriatric assessment in frail ED patients would reduce hospital admissions, lengths of stay (LOS) in hospital, and revisits. Furthermore, coordinating good ED care with rehabilitative measures could avoid functional decline, reduce nursing home admissions and ensure better quality of life.

\section{Methods}

\section{Ethical approval and registration}

This trial complied with the ethical rules stated in the Declaration of Helsinki. The study protocol, informed consent forms, and data protection plan were approved by the Ethics Committee II of the Helsinki University
Hospital (reference number HUS/1711/2018), and the research permit was issued by the Helsinki University Hospital (reference number HUS/278/2018).

The trial was registered in the ClinicalTrials.gov (registration number and date: NCT03751319, 23/11/2018).

\section{Trial design}

This was a single centre, randomised controlled trial with parallel group, two-arm, superiority design. Recruited patients were randomised to the intervention and the control groups with a 1:1 allocation ratio.

\section{Setting}

The trial was conducted in an academic ED with 60000 annual adult patient visits. The ED is adjoined both Helsinki University Hospital and Espoo Community Hospital wards located on the same hospital campus. Older frail patients follow general adult patient pathways in the ED. The ED has a geriatric nurse and physical therapist from adjoining community hospital available on demand, to help with mobilisation and rehabilitative measures and to organise home-care. At the time of trial, the ED had no systematic protocol for geriatric assessment, and no specific geriatric care outside the study protocol was in practice in the ED.

\section{Participants}

The eligibility criteria for participants were the following: an ED-visit during the recruitment period; age $\geq 75$ years; frail, or at risk of frailty, defined by the CFS level of four to nine; and residency in the hospital service area.

\section{Enrolment and randomisation}

A random sequence for two-group allocation was computer-generated using the online service provided by GraphPad Software. No blocking or any restrictions was applied for sequence generation.

Numbered sealed envelopes with corresponding codes inside ("I" for the intervention group and "C" for the control group) were sealed by an assisting person outside the study group.

Screening for eligibility was done at all hours throughout the study period. The ED secretary assigned an individual code for all patients who met the age and residency criteria, and coded research forms were delivered to patients' nurses in the ED. Nurses were asked to assess the CFS-grade of the patient, and to obtain 
background and National Early Warning Score 2 (NEWS2) data if the assessed CFS of the patient was from four to nine. NEWS2 is a structured acute care risk assessment tool based on measured vital signs [26]. Patient enrolment was active during office hours. For eligible patients, written and verbal information about the study was given and consent requested. Consent was obtained from the patient's relative or caregiver in the ED or through a phone call for those who were not capable of consenting. Eligible consented patients were then enrolled and registered in the study. A health-related quality of life (HRQoL) assessment (the EQ-5D-5 Lquestionnaire) was filled in with each patient or with a caregiver, as appropriate. After enrolment, a study physician opened the envelope concordant with the patient's registration number and filled in the study group in the registration sheet. If a major concern or life-threatening condition was recognised during trial enrolment, it was communicated to the ED team providers.

ED team (ED physician of any speciality and ED nurses) treated both intervention and control group patients according to standard principles for chief complaint and acute condition. No systematic assessment or screening for geriatric conditions or further assessment or care were included in standard care, but if ED team recognised a need for geriatric nurse and physical therapist consultations, it was available.

\section{Intervention procedures}

For the intervention group, systematic geriatric assessment was added to their standard care. Assessment was provided separate from ED team care. While the ED team physician was in charge of the acute care for all enrolled patients, the geriatric assessment was led by second physician assigned for the assessment protocol. Two geriatricians and two emergency physicians with consultation support from a geriatrician were allocated for providing the assessment for the six-month study enrolment period. Assessment was performed in structured form, but with consideration of patient co-operation and abilities for each test.

The study physicians based the evaluation of functioning on activities of daily living and observed the patients' ability to walk. An orthostatic test was performed when possible. The Abbreviated Mental Test 4 (AMT4) and Six-Item-Screener were used for assessment of cognitive status $[27,28]$. The study physicians screened the patients for delirium with the 4 'A's Test (4AT) [29], and for depression with the Patient Health Questionnaire 2 (PHQ-2) [30]. Risk of falls and sarcopenia were evaluated using clinical judgment. Medication reviews were performed with special attention to potential adverse effects, interactions, or undertreatment. Any need for further assessment, testing, or rehabilitation was evaluated. The study physicians interviewed the relatives or caregivers for those patients who could not provide a patient history, with the patient's consent as appropriate. If the patient could not co-operate, assessments were performed as feasible, and further information was sought from the electronic medical records (EMR).

After completing the assessments, the study physicians provided individual, multi-dimensional recommendations and advice regarding medical care, medications, rehabilitation, nutrition, and evaluation of the need for home-care to the patient, the ED-physician and nurses providing acute care, hospital wards, and home-care. This was done verbally both in the ED and through phone calls, and with structured documentation in the EMR. A Geriatric nurse and physical therapist from community hospital helped to organise support, care, and at-home rehabilitation for discharged patients. Detailed recommendations of mobilisation, nutrition, further assessments, and medical treatment were forwarded to the hospital wards if the patient was admitted. The protocol for trial participants is summarised in Fig. 1.

\section{Blinding}

Due to the active intervention provided in the ED, blinding of patients or personnel was not possible. However, the study physicians did not actively inform the ED personnel providing care for control patients about the patients' enrolment in the trial. Geriatric assessment and recommendations were documented in the EMR for patient's future care, so outcome-assessment was not blinded.

\section{Outcomes}

The pre-specified primary outcome measure was the cumulative LOS, as given by the total number of overnight stays in hospital wards (both at the tertiary hospital and the community hospital ward) during 365-day follow-up from the date of the enrolment. All hospital admissions, from the index ED-visit or later, during the follow up period were included. Primary outcome data were retrieved from the tertiary hospital EMR with portal to the community hospital records after follow-up.

The pre-specified secondary outcomes were cumulative number of admissions to hospital wards during the 365day follow-up time, admission rate from the index visit, readmissions to the ED within $72 \mathrm{~h}, 30$ days, and 365 days after the index visit, proportion of patients alive and home-dwellingat 365 days after the enrolment, and HRQoL-measurement using the EuroQol EQ-5D-5 L instrument $[31,32]$. Secondary outcome data were acquired by reviewing the EMR, and by phone interview (with patients, or their caregiver as surrogate) for EQ-5D-5 L with standardised forms designed for phone interview, after the 365-day follow-up time. The EuroQol Crosswalk Index Value Calculator v2 with the Denmark value set was used for EQ-5D-5 L index-value calculations. 


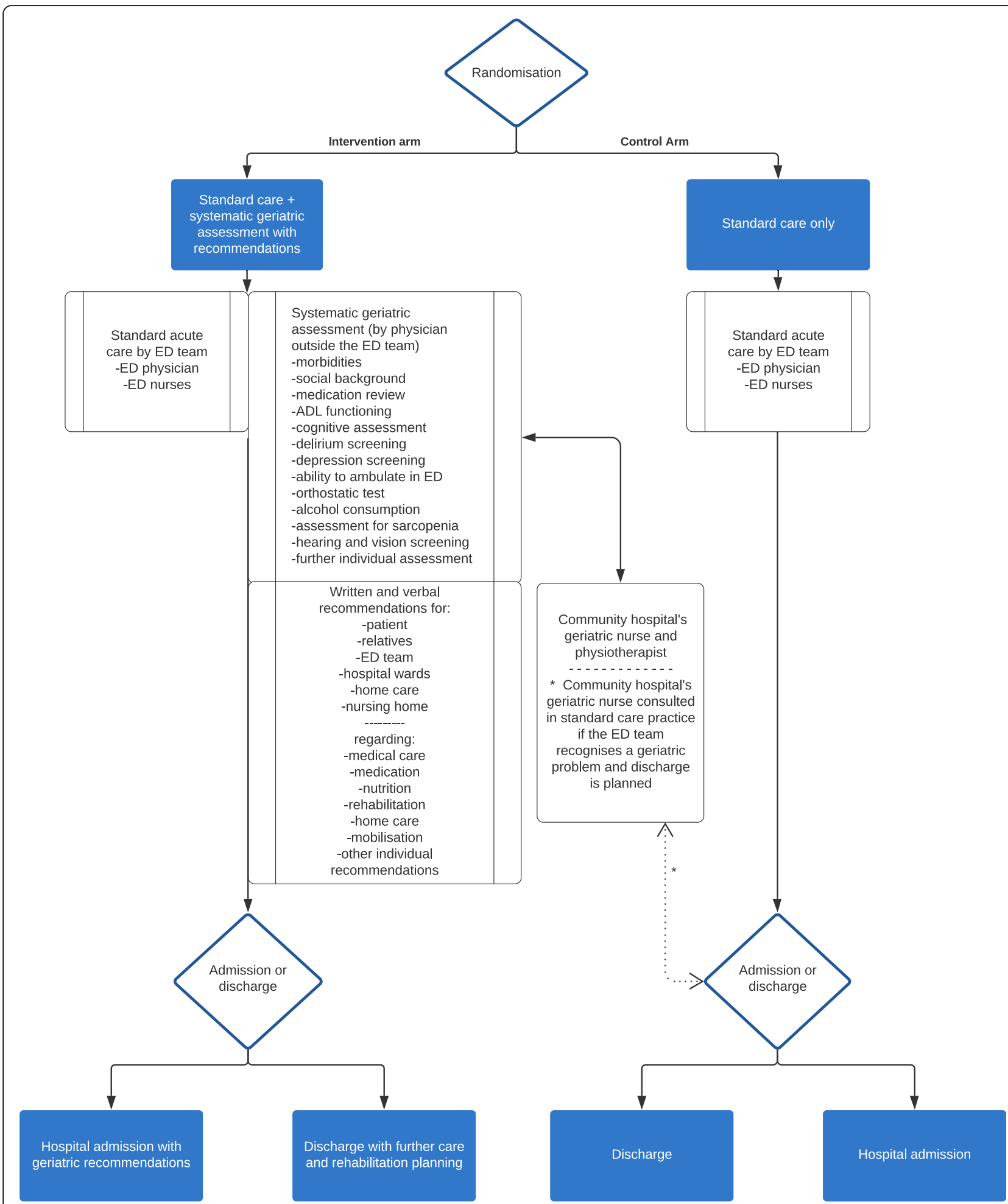

Fig. 1 Flowchart of care in the emergency department for trial participants. Abbreviations: ED, emergency department; ADL, Activities of daily living

The pre-specified outcome measures for assessing the safety and feasibility of the intervention were LOS in the ED on the index-visit, hospital-LOS from the index-visit, 365-day mortality, and number of ED visits related to falls during the follow-up period. Data for these outcomes were obtained by reviewing the EMR. Other prespecified adverse events were not defined. 


\section{Sample size}

We based the sample size calculation on an assumed total hospital stay of $25 \pm 15$ days during the 365-day follow-up time. The assumed hospital stay was an estimation based on previous five-year ED patient data and national reports on inpatient care. A reduction of $10 \%$ in hospital stay was assumed for the intervention group. We calculated a minimum sample size of 392 patients in each group to reach a power of $80 \%$ with $5 \%$ alpha level. A goal was set to recruit a total of 900 patients in order to exceed minimum sample size requirements.

\section{Statistical methods}

For the outcomes depending on 365-day follow-up time, to account for patients who died during the follow-up, total follow-up time contributed by patients in each group were calculated. Rate per 100 person-years and rate ratio estimate with $95 \%$ confidence interval (CI) applying the Byar method were calculated. For the binary outcomes, risk ratios (RR) with $95 \%$ CI were calculated, and the significance of differences were tested with $\chi^{2}$ tests. For the other outcomes, means with standard deviations (SD) and differences of means with $95 \% \mathrm{CI}$, or medians with inter-quartile range (IQR) and difference of medians were calculated. Significance of mean differences were tested with $t$ test and median difference with Mann Whitney U test. A $P$ value $<0.05$ was considered statistically significant for all outcomes. Intentionto-treat analyses were performed, except for the EQ-5D5 L-outcome, where only patients with data available were included.

We used the IBM SPSS Statistics for Windows software version 25.0 (Armonk, NY: IBM Corp) for statistical analyses. OpenEpi, Version 3 calculator was used for rate ratio calculations.

\section{Results \\ Participants}

Patients were enrolled into the trial in a planned sixmonth period between December 11, 2018 and June 7, 2019. We were unable to enrol the anticipated sample size during the planned time period due to slow recruitment. A total of 4356 patients were identified as eligible for the study based on demographics (Fig. 2). The CFS was assessed for 2388 patients, with 1711 patients assessed as within the eligible CFS-class of four to nine. Of those available during office hours, 506 patients were asked, or their caregiver were reached for, for informed consent, of whom 23 patients (or caregivers) declined, and for 42 patients who were not capable for consenting a caregiver was not available. Randomisation was performed for 441 patients. After enrolment, two patients from the intervention group and seven patients from the control group were excluded (enrolled twice or did not meet inclusion criteria). Ultimately, 213 and 219 patients in the intervention and control groups, respectively, were included in the analysis.

Baseline characteristics for the trial groups are described in Table 1 . The median age was 85 (IQR 80-90), the proportion of female patients was $62.7 \%$, the median CFS was 6 (IQR 5-6), and the proportion of patients living at home (with or without home-care assistance) was $89.7 \%$.

We reviewed the EMR after a 365-day follow-up time for each patient. Survival data were available for all patients. Available data related to hospital stay, mortality, admissions, and home-dwelling status were reviewed for all patients.

\section{Intervention}

In the intervention group, all 213 patients were assessed. For two patients, the assessment was interrupted by admission or discharge, but recommendations were given based on available information.

The study physicians gave and documented at least one recommendation for 202/213 (94.8\%) patients. The most frequent recommendation was about medication (given to $168 / 213 ; 78.9 \%$ of the patients), followed by advice on organising home care or rehabilitation-athome services $(65 / 213 ; 30.5 \%)$. Clinical findings and recommendations based on assessment are described in Table 2 .

In the control group, two patients received documented geriatric advice related to a major concern noticed during enrolment. These patients were included in the analysis.

\section{Primary outcome}

Patients in the intervention group had cumulative hospital stay of total 6093 days and contributed follow-up time of total 175.6 person-years when mortality during the follow-up was taken into account. Patients in the control group had cumulative hospital stay of total 5734 days and contributed follow-up time of total 182.1 person-years. Hospital stay rate during the 365-day follow up per 100 person-years for the intervention and control group were: 3470 and 3149 days, respectively, with rate ratio of 1.10 (95\% CI $0.55-2.19)$, without statistically significant difference $(P=.78)$. (Table 3$)$.

\section{Secondary outcomes}

The proportion of hospital admissions from index visit was $7.5 \%$ point lower in the intervention group (133/ $213 ; 62.4 \%)$ than in the control group (153/219; 69.9\%), but without statistical significance, RR 0.89 (95\% CI, $0.78-1.02 ; P=.10)$. During the 30 -day follow-up, means of number of hospital admissions for the intervention 


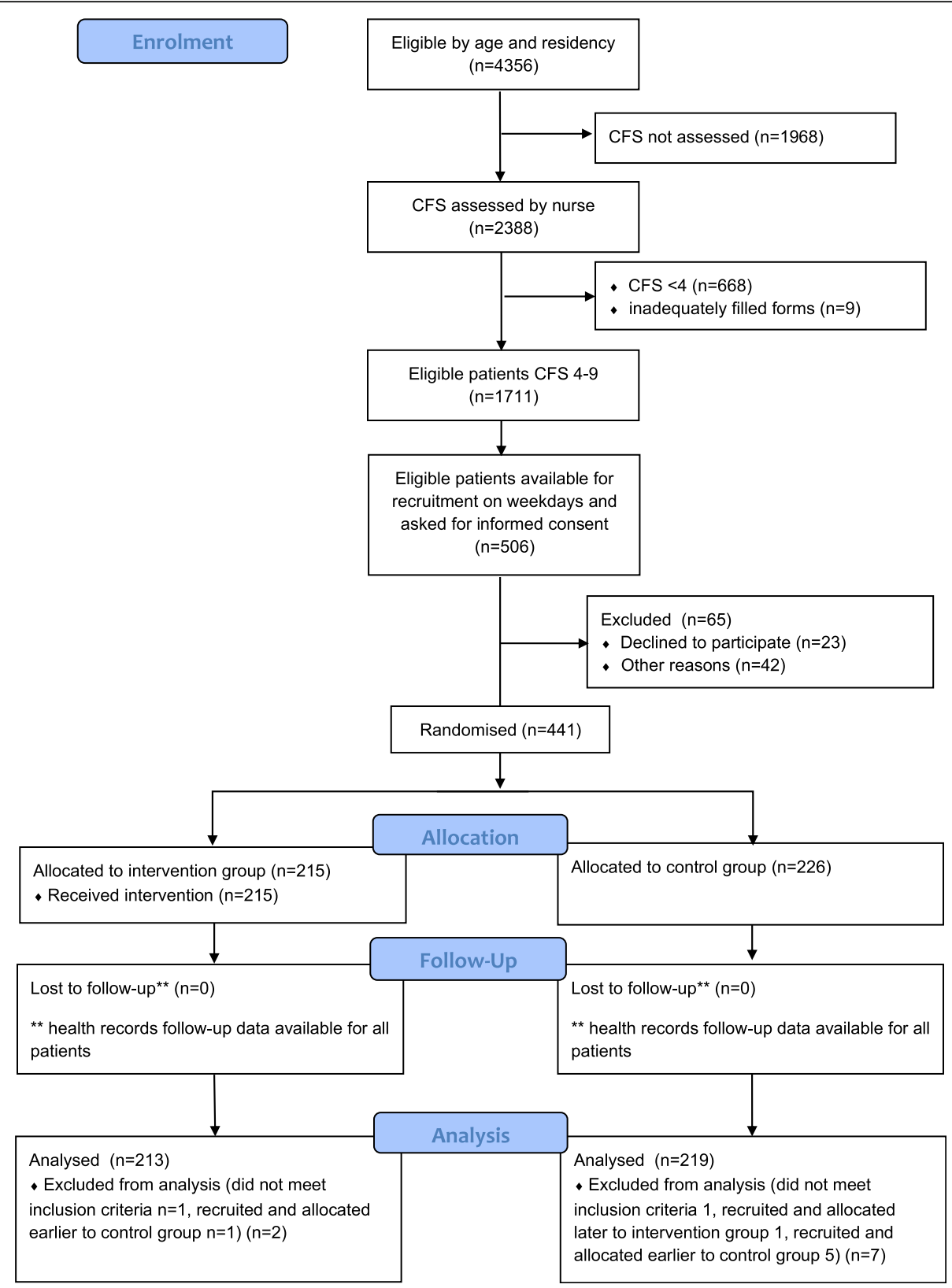

Fig. 2 CONSORT 2010 Flow Diagram of enrolment, intervention allocation, follow-up, and data analysis. Abbreviations: CFS, Clinical frailty scale

and the control group were similar: 0.79 (SD 0.60) and 0.91 (SD 0.64), respectively, with mean difference of -0.12 (95\% CI, $-0.23-0.00 ; P=.48$ ). During the 365-day follow-up hospital admission rates did not differ significantly: 230 admissions per 100 person-years in the intervention group and 246 in the control group, with rate ratio of 0.94 ( $95 \% \mathrm{CI}, 0.82-1.07 ; P=.35$ ).

Readmissions to the ED for the intervention and control group did not significantly differ. In the 72-hour and 30-day follow-up number of ED-readmissions had means of 0.04 (SD 0.19) vs. 0.05 (SD 0.21), difference 0.01 (95\% CI, $-0.05-0.03, P=.40$ ); and 0.29 (SD 0.58) vs.
0.29 (SD 0.54), difference 0.00 (95\% CI, $-0.11-0.11, P=$ $.82)$, respectively. Rates of ED readmission in 365-day follow-up per 100 person-years were: 268 and 238 for the intervention and control group, respectively, with rate ratio of 1.12 (95\% CI, 0.99-1.28), $P=.08$.

The proportions of patients alive and living at home at the end of 365-day follow-up were: 116/213 (54.5\%) in the intervention group, and 111/219(50.7\%) in the control group; RR 1.07 (95\% CI, 0.90-1.29; $P=.43$ ).

A total of 300 EQ-5D-5 L-index follow-up assessments were completed, of which 186 answered by patients and 114 by caregivers. The EQ-5D-5 L-VAS question was 
Table 1 Baseline characteristics of the participants

\begin{tabular}{|c|c|c|c|}
\hline & & Intervention group & Control group \\
\hline & & 213 & 219 \\
\hline \multirow[t]{2}{*}{ Age } & median (IQR) & $85(80-89.5)$ & $85(80-90)$ \\
\hline & range & $75-103$ & $75-102$ \\
\hline Female gender & n (\%) & $136(63.8)$ & $135(61.6)$ \\
\hline CFS & median (IQR) & $6(5-6)$ & $5(5-6)$ \\
\hline Home-dwelling ${ }^{a}$ & n (\%) & $194(91.1)$ & $170(88.1)$ \\
\hline Number of medications ${ }^{b}$ & median (IQR) & $10(7-14)$ & $10(7-14)$ \\
\hline Cardiovascular disease & n (\%) & $123(57.7)$ & $131(59.8)$ \\
\hline Cognitive disorder & n (\%) & $82(38.5)$ & $93(42.5)$ \\
\hline Pulmonary disease & n (\%) & $43(20.2)$ & $46(21.0)$ \\
\hline Malignancy & n (\%) & $28(13.1)$ & $26(11.9)$ \\
\hline Diabetes & n (\%) & $54(25.4)$ & $50(22.8)$ \\
\hline Stroke or TIA in history & n (\%) & $36(16.9)$ & $39(17.8)$ \\
\hline ED chief complaint: trauma & n (\%) & $41(19.2)$ & $43(19.6)$ \\
\hline ED chief complaint: acute condition nontrauma & n (\%) & $166(77.9)$ & $172(78.5)$ \\
\hline ED admission for other or nonspecific cause & n (\%) & $6(2.8)$ & $4(1.8)$ \\
\hline NEWS2 ${ }^{c}$ & median (IQR) & $1(0-3)$ & $1(0-4)$ \\
\hline EQ-5D-5L-index ${ }^{d}$ & mean (SD) & $0.557(0.283)$ & $0.545(0.264)$ \\
\hline EQ-5D-5L-VAS & mean (SD) & $45.4(27.1)$ & $41.8(27.0)$ \\
\hline
\end{tabular}

Abbreviations: IQR interquartile range, SD standard deviation, NEWS2 National Early Warning Score 2, ED emergency department, VAS visual analog scale ${ }^{a}$ Home-dwelling status data acquired during the intervention included for the intervention group. Data available for 213 patients in the intervention group and 193 patients in the control group

${ }^{b}$ Number of medications data available for 108 patients in the intervention group and 119 in the control group

CNEWS2 data of ED admission available for 180 patients in the intervention group and 193 in the control group

${ }^{d} E Q-5 D-5 L-i n d e x$-data available for 112 patients in the intervention group and 129 in the control group

EQ-5D-5L-VAS -data available for 118 patients in the intervention group and 139 in the control group

answered for 299 patients. The EQ-5D-5 L-index for the intervention and the control group after 365-day followup were mean 0.53 (SD 0.28) and 0.53 (SD 0.27), respectively (difference $0.00,95 \% \mathrm{CI},-0.06-0.06 ; P>.99$ ), and the EQ-5D-5 L-VAS values were mean 55.3 (SD 23.8 ) and 53.2 (SD 0.22.8), respectively (difference 2.0; $95 \%$ CI, $-3.3-7.4 ; P=.45)$.

The ED-LOS between the groups did not significantly differ: median 7:23 h:minutes (IQR 5:22 - 15:23) and 9: $25 \mathrm{~h}$ :minutes (IQR 6:00-18:40), for the intervention and the control groups, respectively $(P=.05)$. Hospital-LOS for patients admitted from the index-ED-visit were: mean 15.91 (SD 24.30) days for the intervention group and 12.30 (20.38) for the control group, mean difference 3.61 (95\% CI, -1.67-8.83), $P=.07$. The 30-day and 365day mortalities were similar between the groups: 30-day mortality was $16 / 213(7.5 \%)$ for the intervention group, and 15/219 (6.8) for the control group, RR 1.10 (95\% CI, $0.56-2.16 ; P=.79)$, and 365 -day mortality was $53 / 213$ (24.9\%) for the intervention group, and 59/219 (26.9\%) for the control group, RR $0.92(95 \% \mathrm{CI}, 0.67-1.27 ; P=$ .63). Rates per 100 person-years for ED visit related to falls during 365-day follow-up were: 59 for the intervention group and 47 for the control group, with rate ratio of 1.27 (95\% CI, $0.95-1.69 ; P=.10)$.

\section{Discussion}

In this study, the anticipated reduction in hospital stay was not achieved with systematic geriatric assessment provided in the ED. Hospital admission rate from the index visit was lower in the intervention group without an increased number of ED-revisits, but without statistical significance. There was no significant difference in the ED-LOS, hospital-LOS after the index visit, hospital admission rates, 365-day mortality, ED-revisits for falls, home-dwelling status, or HRQoL at the end of the follow-up, between the intervention and the control groups.

There are many possible explanations for the null results. First, study patients were heterogeneous with some of them possibly having no chance of benefiting from the intervention due to, for example, terminal illness. However, most patients were home-dwelling at baseline with multi-morbidity and impairment in daily functions, so it can be argued that the patient population in this trial should benefit from the intervention. As yet, no 
Table 2 Assessment and recommendations in the intervention group

\begin{tabular}{|c|c|c|}
\hline Intervention group & $\mathrm{n}$ & 213 \\
\hline \multirow[t]{2}{*}{ AMT-4 } & n (\%) & $188(88.3)$ \\
\hline & median (IQR) & $4(2-4)$ \\
\hline 4 & n (\%) & 106/188 (56.4) \\
\hline 3 & n (\%) & $32 / 188(17.0)$ \\
\hline 2 & n (\%) & 24/188 (12.8) \\
\hline 1 & n (\%) & 14/188 (7.4) \\
\hline 0 & n (\%) & 11/188 (5.9) \\
\hline \multirow[t]{2}{*}{ Six-Item-Screener } & n (\%) & $153(71.8)$ \\
\hline & median (IQR) & $4(2-6)$ \\
\hline \multirow[t]{2}{*}{ 4AT } & n (\%) & $186(87.3)$ \\
\hline & median (IQR) & $1(0-3)$ \\
\hline PHQ-2 & n (\%) & $147(69.0)$ \\
\hline $1-2$ & n (\%) & $60 / 147(40.8)$ \\
\hline 0 & n (\%) & $87 / 147(59.2)$ \\
\hline Orthostatic test & n (\%) & $60(28.2)$ \\
\hline orthostatic hypotension & n (\%) & 20/60 (33.3) \\
\hline Alcohol consumption & n (\%) & $189(88.7)$ \\
\hline alcohol use (any) & n (\%) & $35 / 189(18.5)$ \\
\hline Sarcopenia or cachexia & n (\%) & $209(98.1)$ \\
\hline sarcopenia or cachexia present & n (\%) & $89 / 209(42.6)$ \\
\hline Falls in last 12 months & n (\%) & $161(75.6)$ \\
\hline 2 or more & n (\%) & $84 / 161(52.2)$ \\
\hline 1 & n (\%) & 24/161 (14.9) \\
\hline 0 & n (\%) & $53 / 161(32.9)$ \\
\hline Recommendations or advice & n (\%) & $213(100.0)$ \\
\hline Any & n (\%) & $202(94.8)$ \\
\hline Medication & n (\%) & $168(78.9)$ \\
\hline Home support services $^{a}$ & n (\%) & $65(30.5)$ \\
\hline Support or rehabilitation for mobility & n (\%) & $58(27.2)$ \\
\hline Dietary & n (\%) & $49(23)$ \\
\hline
\end{tabular}

Abbreviations: AMT-4 "Abbreviated Mental Test 4", 4AT "4 A'2s test for Delirium Screening", PHQ-2 "Patient Health Questionnaire-2: Screening Instrument for Depression"

a home care, rehabilitation-at-home, further assessment for supportive care

adapted, more specific criteria exist for targeting CGA for patients in the ED. It is challenging to find efficient interventions for this heterogeneous patient population in the complex environment of emergency care.

Second, the deviance in the cumulative hospital stay was large, and only a small proportion of patients contributed the major proportion of the hospital stay ( $42 \%$ of total hospital-stay days of the study population were occupied by patients in the highest decile in cumulated hospital stay). It is possible that home-care-based implementation of care and rehabilitation are insufficient for patients who are vulnerable to prolonged hospital stay.
Third, patients in this study were cared for in many different organisations in the tertiary hospital, community hospital, home care, outpatient clinics and nursing homes. Delivering recommendations to patients and other institutions in written and verbal form was emphasised in the intervention, but it is not known how thoroughly these recommendations were implemented.

Fourth, standard treatment in EDs probably varies according to how well geriatric syndromes and frailtyrelated issues are taken into account, and how geriatric care is organised. In the ED of this study, no systematic assessment protocol for geriatric problems existed in standard care, but awareness of good geriatric care may have been prevalent, and many services from community hospital including geriatric nurse were available for control group patients if need for such service was recognised. Therefore, some dilution of the efficacy may have occurred.

Identifying frail patients in hospital wards remain crucial for early initialisation of hospital-based-CGA, which has been shown to be efficient [11]. Therefore, assessment for frailty status in the ED may be a route towards more comprehensive care, but, compared to intervention in this trial, more intensive and continuous ward-based interventions may be needed for admitted patients. Efficient interventions remain unknown for those frail patients who are discharged from the ED. Similar results to this trial were reported in a randomised trial in which CGA was provided after discharge from acute medical units [33].

In previous, nonrandomised studies, the admission rate of older patients from ED was reduced by EDrelated CGA by $2.6-19.7 \%$ points [34]. In our study, patients in the intervention group were less often admitted to hospital, with no difference in ED-revisit rate. The reduction of admission rate did not reach statistical significance here, but, in the light of previous studies, the efficacy of intervention for this outcome is likely to be real. For admitted patients, the hospital-LOS was longer. The intervention might have altered patient selection for admission, increasing the hospital LOS. It is also possible that recommended further assessments and interventions contributed for longer LOS. Assuming that unnecessary hospital admissions can be safely avoided with geriatric interventions in the ED, it remains to be demonstrated whether this effect prevails in a longer period when the same patients have multiple ED-visits. Considering the risk of hospitalisation-acquired disability, promoting discharge for patients who do not have an absolute need for hospital care is likely to be beneficial for the patients [35].

Overall, the findings of our study concur with late reviews assessing ED-based interventions for older adults [25, 36]. Individualised, more effective geriatric interventions for frail 
Table 3 Clinical outcomes of the trial

\begin{tabular}{|c|c|c|c|c|c|}
\hline Outcome & & $\begin{array}{l}\text { Intervention } \\
n=213\end{array}$ & $\begin{array}{l}\text { Control } \\
n=219\end{array}$ & ratio or difference $[95 \% \mathrm{Cl}]$ & $p$ value \\
\hline \multicolumn{6}{|l|}{ Primary outcome } \\
\hline $\begin{array}{l}\text { Cumulative hospital stay(days) during } \\
365 \mathrm{~d} \text { follow-up }\end{array}$ & $\begin{array}{l}\text { rate per } 100 \\
\text { person-years }\end{array}$ & 3470 & 3149 & rate ratio $1.10[0.55 ; 2.19]$ & 0.783 \\
\hline \multicolumn{6}{|l|}{ Secondary outcomes } \\
\hline Admission from ED index visit & n (\%) & $133(62.4)$ & $153(69.9)$ & risk ratio 0.89 [0.78;1.02] & 0.103 \\
\hline Number of hospital admissions30d & mean (SD) & $0.79(0.60)$ & $0.91(0.64)$ & mean difference $-0.12[-0.23 ; 0.00]$ & 0.475 \\
\hline Hospital admissions 365d & $\begin{array}{l}\text { rate per } 100 \\
\text { person-years }\end{array}$ & 230 & 246 & rate ratio $0.94[0.82 ; 1.07]$ & 0.345 \\
\hline Number of ED readmissions $72 \mathrm{~h}$ & mean (SD) & $0.04(0.19)$ & $0.05(0.21)$ & mean difference $-0.01[-0.05 ; 0.03]$ & 0.400 \\
\hline Number of ED readmissions 30d & mean (SD) & $0.29(0.58)$ & $0.29(0.54)$ & mean difference $0.00[-0.11 ; 0.11]$ & 0.817 \\
\hline ED readmissions $365 d$ & $\begin{array}{l}\text { rate per } 100 \\
\text { person-years }\end{array}$ & 268 & 238 & rate ratio $1.12[0.99 ; 1.28]$ & 0.081 \\
\hline Alive and living-at-home 365d & n (\%) & $116(54.5)$ & $111(50.7)$ & RR $1.07[0.90 ; 1.29]$ & 0.432 \\
\hline EQ-5D-5 L-index $365 d^{a}$ & mean (SD) & $0.53(0.28)$ & $0.53(0.27)$ & mean difference $0.0[-0.06 ; 0.06]$ & 0.995 \\
\hline EQ-VAS $365 \mathrm{~d}^{\mathrm{b}}$ & mean (SD) & $55.28(23.83)$ & $53.23(22.78)$ & mean difference $2.04[-3.26 ; 7.35]$ & 0.449 \\
\hline \multicolumn{6}{|l|}{ Other outcomes } \\
\hline ED LOS hh:mm & median (IQR) & $7: 23(5: 22-15: 23)$ & $9: 25(6: 00-18: 40)$ & median difference - 2:02 & 0.052 \\
\hline $\begin{array}{l}\text { Hospital LOS from the index-visit } \\
\text { if admitted (days) }\end{array}$ & mean (SD) & $15.91(24.30)$ & $12.30(20.38)$ & mean difference $3.61[-1.67 ; 8.83]$ & 0.069 \\
\hline Death 365d & n (\%) & $53(24.9)$ & $59(26.9)$ & risk ratio 0.92 [0.67;1.27] & 0.626 \\
\hline Death 30d & n (\%) & $16(7.5)$ & $15(6.8)$ & risk ratio 1.10 [0.56;2.16] & 0.790 \\
\hline ED visits for falls $365 d^{d}$ & $\begin{array}{l}\text { rate per } 100 \\
\text { person-years }\end{array}$ & 59 & 47 & rate ratio 1.27 [0.95;1.69] & 0.104 \\
\hline
\end{tabular}

Abbreviations: CI Confidence Interval, ED Emergency Department, SD Standard Deviation, LOS Length of Stay, IQR interquartile range

adata available for 150 patients in the intervention group, 150 patients in the control group

${ }^{b}$ data available for 149 patients in the intervention group, and 150 patients in the control group

cOnly patients admitted from the index ED visit included. Only the first hospital stay period counted for LOS.

d patients who visited the ED after the index ED visit for fall-related complaint

patients in the EDs, and detailed identification for patients who benefit from such interventions, remain to be studied.

\section{Strengths and limitations}

This study has many strengths. The randomised, controlled trial protocol enables a robust assessment of the efficacy of the intervention. For the primary outcome, and most of the secondary and other outcomes, reliable outcome data were available from the EMR. The trial protocol was implemented in a real-life environment with typical frail patients in the ED. Furthermore, our mid-sized ED, with its nonselected adult patient population, can be considered a typical acute care setting.

Besides the nonblinded protocol, a major limitation in this trial was that enrolment did not fulfil the expected sample size. An important reason for this was that the enrolment process was more laborious than anticipated. Patients had acute care underway so availability for trial enrolment and intervention often had to wait, slowing recruitment. However, this reflects the real challenges when providing geriatric assessment amidst ongoing acute care, and is a real-life feasibility issue in the ED environment. There was not trend of fewer hospitalstay-days in 365-day follow-up for the intervention group, so the reduced sample size is probably not the reason for the null result for the primary outcome.

The CFS was assessed only for $55 \%$ of patients who met the demographic criteria for the study, probably because nursing staff were occupied due to crowding. Study enrolment was active only during office-hours when a study physician was available. Thus, patients enrolled for the study may not be fully representative of frail older patients who visit the ED in late hours or during weekends.

Hospital-stay data was reviewed for all patients, but it is possible that some patients may have been admitted outside our hospital network. However, in Finland, private in-patient hospital beds outside public health care are negligible in number, and we found no documentation of such admissions in the patient records. Therefore, the hospital admission data can be considered conclusive.

Standard care by ED teams was focused on acute conditions, but it is possible that some "leakage" of 
intervention affected the control group indirectly when personnel was aware of the ongoing trial and may have put more emphasis on geriatric issues in patient care.

\section{Conclusions}

Systematic geriatric assessment and recommendations for older adults with frailty in the ED did not reduce cumulative hospital stay during one-year follow-up. No significant differences were found between the intervention and the control groups in the secondary outcomes of hospital admissions, revisits to the ED, living-at-home status, quality-of-life, or other outcomes.

\section{Abbreviations}

AMT4: Abbreviated mental test 4; AT4: 4 'A's test; CFS: Clinical Frailty Scale; CGA: Comprehensive geriatric assessment; $\mathrm{Cl}$ : Confidence interval; ED: Emergency department; EMR: Electronic medical records; HRQoL: Healthrelated quality of life; IQR: Inter-quartile range; LOS: Length of stay; NEWS2: National early warning score 2; PHQ-2: Patient health questionnaire 2: RR: Risk ratio; SD: Standard deviation

\section{Acknowledgements}

We thank the staff of Jorvi ED for their collaboration in the study; Susanne Lehvonen for helping in the implementation of the study protocol; Pia Harjola for helping in study preparations; Vesa Launiainen for help in patient enrolment and intervention; Elina Lehtelä for data processing; Minna Pajuportti for carrying out the follow-up interviews; Paula Bergman for advising in statistics; and John Weston for language editing.

\section{Authors' contributions}

The authors contributions for the trial are as follows: conception and design: $J A, K K, T S, M C, J T, V H$; acquisition of data: JA, KK, MC, DJ, VH; analysis and interpretation of data: JA, KK, TS, MC, DJ, JT, VH; drafting and approval of the manuscript: JA, KK, TS, MC, DJ, JT, VH.

\section{Funding}

The trial was funded through state funding for university-level health research, granted by the Helsinki University Hospital, and the Department of Emergency Medicine and Services, Helsinki University Hospital. JA and KK received personal grants for the study from the Finnish Association for Emergency Medicine and the Finnish Medical Foundation. The funding bodies were not involved in the design of the study, collection, analysis, or interpretation of the data, or in writing the manuscript.

No sponsors were involved in the trial.

\section{Availability of data and materials}

The datasets generated and analysed during the trial are not publicly available due national juridical restrictions protecting pseudonymised research data. Based on national jurisdiction ethical board or organisation's study permission does not allow sharing pseudonymised trial data. Further description or analysis of data are however available from the authors upon reasonable request.

\section{Declarations}

\section{Ethics approval and consent to participate}

The study protocol, informed consent forms, and data protection plan were approved by the Ethics Committee II of the Helsinki University Hospital (reference number HUS/1711/2018), and the research permit was issued by the Helsinki University Hospital (reference number HUS/278/2018). Written informed consent to participate was obtained from all patients who were considered to be capable for consenting. Informed consent to participate was obtained from the patient's relative or caregiver in the ED or through a phone call for those patients who were considered not capable of consenting for themselves.
All methods were carried out in accordance with relevant guidelines and regulations.

\section{Consent for publication}

Not applicable.

\section{Competing interest}

The authors declare that they do not have any competing interests.

\section{Author details}

${ }^{1}$ Department of Emergency Medicine and Services, University of Helsinki and Helsinki University Hospital, PL 340 |Haartmaninkatu 4, 00029 HUS Helsinki, Finland. ${ }^{2}$ University of Helsinki and Helsinki University Hospital, Helsinki, Finland. ${ }^{3}$ Centre for Life Course Health Research, University of Oulu, Oulu, Finland. ${ }^{4}$ Päijät-Häme Joint Authority for Health and Wellbeing, Services for Older People, Lahti, Finland. ${ }^{5}$ Department of Internal Medicine, University of Helsinki and Helsinki University Hospital, Helsinki, Finland.

Received: 16 March 2021 Accepted: 13 June 2021

Published online: 02 July 2021

\section{References}

1. Theou O, Campbell S, Malone ML, Rockwood K. Older Adults in the Emergency Department with Frailty. Clin Geriatr Med. 2018;34(3):369-86. https://doi.org/10.1016/j.cger.2018.04.003.

2. Brousseau AA, Dent E, Hubbard R, et al. Multinational Emergency Department Study: Identification of older adults with frailty in the Emergency Department using a frailty index: results from a multinational study. Age Ageing. 2018;47(2):242-8. https://doi.org/10.1093/ageing/afx168.

3. Lewis $E T$, Dent $E$, Alkhouri $H$, et al. Which frailty scale for patients admitted via Emergency Department? A cohort study. Arch Gerontol Geriatr. 2019;80: 104-14. https://doi.org/10.1016/j.archger.2018.11.002.

4. Jorgensen R, Brabrand M. Screening of the frail patient in the emergency department: A systematic review. Eur J Intern Med. 2017;45:71-3. https://doi. org/10.1016/j.ejim.2017.09.036.

5. Rockwood K, Song X, Macknight C, et al. A global clinical measure of fitness and frailty in elderly people. CMAJ. 2005;173(5):489-95. https://doi.org/10.1 503/cmaj.050051.

6. Kaeppeli T, Rueegg M, Dreher-Hummel T, et al. Validation of the Clinical Frailty Scale for Prediction of Thirty-Day Mortality in the Emergency Department. Ann Emerg Med. 2020;76(3):291-300. https://doi.org/10.1016/j.a nnemergmed.2020.03.028.

7. Lo AX, Heinemann AW, Gray E, et al. Inter-rater Reliability of Clinical Frailty Scores for Older Patients in the Emergency Department. Acad Emerg Med. 2021;28(1):110-3. https://doi.org/10.1111/acem.13953.

8. Wallis SJ, Wall J, Biram RW, Romero-Ortuno R. Association of the clinical frailty scale with hospital outcomes. QJM. 2015;108(12):943-9. https://doi. org/10.1093/qjmed/hcv066.

9. Cardona M, O'Sullivan M, Lewis ET, et al. Prospective Validation of a Checklist to Predict Short-term Death in Older Patients After Emergency Department Admission in Australia and Ireland. Acad Emerg Med. 2019; 26(6):610-20. https://doi.org/10.1111/acem.13664.

10. Elliott A, Taub N, Banerjee J, et al. Does the Clinical Frailty Scale at Triage Predict Outcomes From Emergency Care for Older People? Ann Emerg Med. 2020;S0196-0644(20):307-9. https://doi.org/10.1016/j.annemergmed.2 020.09.006 [Epub ahead of print].

11. Ellis G, Gardner M, Tsiachristas A, et al. Comprehensive geriatric assessment for older adults admitted to hospital. Cochrane Database Syst Rev. 2017;9: CD006211. https://doi.org/10.1002/14651858.CD006211.pub3.

12. Ellis G, Jamieson CA, Alcorn M, Devlin V. An Acute Care for Elders (ACE) unit in the emergency department. Eur Geriatr Med. 2012;3(4):261-3.

13. Arendts G, Fitzhardinge S, Pronk K, Donaldson M, Hutton M, Nagree Y. The impact of early emergency department allied health intervention on admission rates in older people: a non-randomized clinical study. BMC Geriatr. 2012;12:8. https://doi.org/10.1186/1471-2318-12-8.

14. Conroy SP, Ansari K, Williams M, et al. A controlled evaluation of comprehensive geriatric assessment in the emergency department: the 'Emergency Frailty Unit.' Age Ageing. 2014;43(1):109-14. https://doi.org/10.1 093/ageing/aft087.

15. Wright PN, Tan G, lliffe S, Lee D. The impact of a new emergency admission avoidance system for older people on length of stay and same-day 
discharges. Age Ageing. 2014;43(1):116-21. https://doi.org/10.1093/ageing/a $\mathrm{ft} 086$.

16. Biese KJ, Busby-Whitehead J, Cai J, et al. Telephone Follow-Up for Older Adults Discharged to Home from the Emergency Department: A Pragmatic Randomized Controlled Trial. J Am Geriatr Soc. 2018;66(3):452-8. https://doi. org/10.1111/jgs.15142.

17. Eklund K, Wilhelmson K, Gustafsson H, Landahl S, Dahlin-Ivanoff S. One-year outcome of frailty indicators and activities of daily living following the randomised controlled trial: "Continuum of care for frail older people". BMC Geriatr. 2013:13:76. https://doi.org/10.1186/1471-2318-13-76.

18. Gagnon AJ, Schein C, McVey L, Bergman H. Randomized controlled trial of nurse case management of frail older people. J Am Geriatr Soc. 1999;47(9): 1118-24. https://doi.org/10.1111/j.1532-5415.1999.tb05238.x

19. Hastings $\mathrm{SN}$, Heflin MT. A systematic review of interventions to improve outcomes for elders discharged from the emergency department. Acad Emerg Med. 2005;12(10):978-86. https://doi.org/10.1197/j.aem.2005.05.032.

20. McCusker J, Verdon J, Tousignant P, de Courval LP, Dendukuri N, Belzile E. Rapid emergency department intervention for older people reduces risk of functional decline: results of a multicenter randomized trial. J Am Geriatr Soc. 2001;49(10):1272-81. https://doi.org/10.1046/j.1532-5415.2001.49254.x.

21. Miller DK, Lewis LM, Nork MJ, Morley JE. Controlled trial of a geriatric casefinding and liaison service in an emergency department. J Am Geriatr Soc. 1996;44(5):513-20. https://doi.org/10.1111/j.1532-5415.1996.tb01435.x.

22. Mion LC, Palmer RM, Meldon SW, et al. Case finding and referral model for emergency department elders: a randomized clinical trial. Ann Emerg Med. 2003;41(1):57-68. https://doi.org/10.1067/mem.2003.3.

23. Caplan GA, Williams AJ, Daly B, Abraham K. A randomized, controlled trial of comprehensive geriatric assessment and multidisciplinary intervention after discharge of elderly from the emergency department-the DEED II study. J Am Geriatr Soc. 2004;52(9):1417-23. https://doi.org/10.1111/j.1532-5415.2 004.52401.X.

24. Arendts $\mathrm{G}$, Bullow K, Etherton-Beer $\mathrm{C}$, et al. A randomized-controlled trial of a patient-centred intervention in high-risk discharged older patients. Eur J Emerg Med. 2018;25(4):237-41. https://doi.org/10.1097/MEJ. 0000000000000444.

25. Hughes JM, Freiermuth CE, Shepherd-Banigan M, et al. Emergency Department Interventions for Older Adults: A Systematic Review. J Am Geriatr Soc. 2019:67(7):1516-25. https://doi.org/10.1111/jgs.15854.

26. Pimentel MAF, Redfern OC, Gerry $\mathrm{S}$, et al. A comparison of the ability of the National Early Warning Score and the National Early Warning Score 2 to identify patients at risk of in-hospital mortality: a multi-centre database study. Resuscitation. 2019;134:147-56. https://doi.org/10.1016/j.resuscita tion.2018.09.026

27. Swain DG, Nightingale PG. Evaluation of a shortened version of the Abbreviated Mental Test in a series of elderly patients. Clin Rehabil. 1997; 11(3):243-8. https://doi.org/10.1177/026921559701100308.

28. Callahan CM, Unverzagt FW, Hui SL, Perkins AJ, Hendrie HC. Six-item screener to identify cognitive impairment among potential subjects for clinical research. Med Care. 2002;40(9):771-81. https://doi.org/10.1097/ 00005650-200209000-00007.

29. Bellelli G, Morandi A, Davis DH, et al. Validation of the 4AT, a new instrument for rapid delirium screening: a study in 234 hospitalised older people. Age Ageing. 2014;43(4):496-502. https://doi.org/10.1093/ageing/a fu021.

30. Kroenke K, Spitzer RL, Williams JB. The Patient Health Questionnaire-2: validity of a two-item depression screener. Med Care. 2003;41(11):1284-92. https://doi.org/10.1097/01.MLR.0000093487.78664.3C.

31. Herdman M, Gudex C, Lloyd A, et al. Development and preliminary testing of the new five-level version of EQ-5D (EQ-5D-5L). Qual Life Res. 2011;20(10): 1727-36. https://doi.org/10.1007/s11136-011-9903-x.

32. Janssen MF, Pickard AS, Golicki D, et al. Measurement properties of the EQ5D-5L compared to the EQ-5D-3L across eight patient groups: a multicountry study. Qual Life Res. 2013;22(7):1717-27. https://doi.org/10.1007/ s11136-012-0322-4

33. Edmans J, Bradshaw L, Franklin M, Gladman J, Conroy S. Specialist geriatric medical assessment for patients discharged from hospital acute assessment units: randomised controlled trial. BMJ. 2013;347:f5874. https://doi.org/1 $0.1136 / \mathrm{bmj} . f 5874$.

34. Jay S, Whittaker P, McIntosh J, Hadden N. Can consultant geriatrician led comprehensive geriatric assessment in the emergency department reduce hospital admission rates? A systematic review. Age Ageing. 2017;46(3):36672. https://doi.org/10.1093/ageing/afw231.

35. Covinsky KE, Pierluissi E, Johnston CB. Hospitalization-associated disability: "She was probably able to ambulate, but I'm not sure". JAMA. 2011;306(16): 1782-93. https://doi.org/10.1001/jama.2011.1556.

36. Harding S. Comprehensive geriatric assessment in the emergency department. Age Ageing. 2020;49(6):936-8. https:/doi.org/10.1093/ageing/afaa059.

\section{Publisher's Note}

Springer Nature remains neutral with regard to jurisdictional claims in published maps and institutional affiliations.
Ready to submit your research? Choose BMC and benefit from:

- fast, convenient online submission

- thorough peer review by experienced researchers in your field

- rapid publication on acceptance

- support for research data, including large and complex data types

- gold Open Access which fosters wider collaboration and increased citations

- maximum visibility for your research: over $100 \mathrm{M}$ website views per year

At $\mathrm{BMC}$, research is always in progress.

Learn more biomedcentral.com/submissions 